\title{
BIFURCATION OF LIMIT CYCLES FROM A 2-DIMENSIONAL CENTER INSIDE $\mathbb{R}^{n}$
}

\author{
JAUME LLIBRE AND AMAR MAKHLOUF
}

\begin{abstract}
We study the bifurcation of limit cycles from the periodic orbits of a linear differential system in $\mathbb{R}^{n}$ perturbed inside a class of piecewise linear differential systems, which appears in a natural way in control theory. Our main result shows that at most one limit cycle can bifurcate up to first order expansion of the displacement function with respect to the small parameter. This upper bound is reached. For proving this result we use the averaging theory in a form where the differentiability of the system is not needed.
\end{abstract}

\section{INTRODUCTION}

Piecewise linear differential systems appear in a natural way in control theory, see for instance $[1,2,3,8,11,14,16]$. These systems can present the complicated dynamical phenomena as the general nonlinear differential systems. One of the main ingredients in the qualitative description of the dynamical behavior of a differential system is the number and distribution of the limit cycles.

The goal of this paper is to study in $\mathbb{R}^{n}$ for all $n \geq 2$ the existence of limit cycles of the control system of the form

$$
\dot{x}=A_{0} x+\varepsilon F(x),
$$

for $|\varepsilon| \neq 0$ a sufficiently small real parameter, where

$$
A_{0}=\left(\begin{array}{ccccc}
0 & -1 & 0 & \cdots & 0 \\
1 & 0 & 0 & \cdots & 0 \\
0 & 0 & 0 & \cdots & 0 \\
\vdots & \vdots & \vdots & \vdots & \vdots \\
0 & 0 & 0 & \cdots & 0
\end{array}\right),
$$

and $F: \mathbb{R}^{n} \rightarrow \mathbb{R}^{n}$ is given by

$$
F(x)=A x+\varphi\left(k^{T} x\right) b,
$$

1991 Mathematics Subject Classification. Primary 34C05, 34A34, 34C14.

Key words and phrases. limit cycles, bifurcation, control systems, averaging method.

The first author is partially supported by a DGICYT/FEDER grant number MTM2008-03437 and by a CICYT grant number 2005SGR 00550. 
with $A \in \mathcal{M}_{n}(\mathbb{R}), k, b \in \mathbb{R}^{n} \backslash\{0\}$ and $\varphi: \mathbb{R} \rightarrow \mathbb{R}$ the piecewise linear function

$$
\varphi(x)= \begin{cases}-1, & \text { for } x \in(-\infty,-1) \\ x, & \text { for } x \in[-1,1] \\ 1, & \text { for } x \in(1, \infty)\end{cases}
$$

The independent variable is denoted by $t$, vectors of $\mathbb{R}^{n}$ are column vectors, and $k^{T}$ denotes the transposed vector.

In the next lemma we show that, through a linear change of variables, it is possible to reduce the number of parameters and take $k=e_{1}$, where $e_{1}^{T}=(1,0, \ldots, 0) \in \mathbb{R}^{n}$.

Lemma 1. Assume that $k_{1}^{2}+k_{2}^{2} \neq 0$ if $n=2$ and that $\left(k_{1}^{2}+k_{2}^{2}\right) k_{3} \neq 0$ if $n>2$. Then by a linear change of variables system (1) can be transformed into the system

$$
\dot{x}=A_{1} x+\varepsilon \bar{A} x+\varepsilon \varphi\left(x_{1}\right) \bar{b},
$$

where $\bar{A} \in \mathcal{M}_{n}(\mathbb{R})$ and $\bar{b} \in \mathbb{R}^{n}$ are convenient functions of $A$ and $b$, and

$$
\begin{gathered}
A_{1}=\left(\begin{array}{cc}
0 & -1 \\
1 & 0
\end{array}\right) \quad \text { if } n=2, \text { and } \\
A_{1}=\left(\begin{array}{cccccc}
0 & -1 & \varepsilon & 0 & \ldots & 0 \\
1 & 0 & \varepsilon & 0 & \ldots & 0 \\
0 & 0 & 0 & 0 & \ldots & 0 \\
\vdots & \vdots & \vdots & \vdots & \ldots & \vdots \\
0 & 0 & 0 & 0 & \ldots & 0
\end{array}\right) \quad \text { if } n>2 .
\end{gathered}
$$

Lemma 1 is proved in Section 3.

For $\varepsilon=0$ system (1) or (3) becomes

$$
\dot{x}_{1}=-x_{2}, \quad \dot{x}_{2}=x_{1}, \quad \dot{x}_{3}=0, \quad \ldots, \quad \dot{x}_{n}=0 .
$$

We note that the origin of every plane of the form $x_{k}=$ constant for $k=$ $3, \ldots, n$ is a global isochronous center for (4), i.e. all the orbits contained in such a plane different from the origin are periodic with the same period $2 \pi$.

A limit cycle is an isolated periodic orbit in the set of periodic orbits. The Poincaré mapping (or, equivalently, the displacement map) is a suitable tool for studying limit cycles of autonomous systems (detailed explanations can be found in $[7,9,10]$, see also Section 2). We remind that a limit cycle of some system corresponds to an isolated zero of the displacement function. Our main result is the following.

Theorem 2. For $n \geq 2$ at most one limit cycle of system (3) bifurcates from the periodic orbits of system (4), up to first order expansion of the displacement function of (3) with respect to the small parameter $\varepsilon$. More precisely if 
(i) the determinant of the minor of the matrix $\bar{A}=\left(a_{i j}\right)$ erasing the first two rows and the first two columns is not zero (of course this condition only is required if $n>2)$, and

(ii) $\left(a_{11}+a_{22}+b_{1}\right) / b_{1} \in(0,1)$ where $\bar{b}=\left(b_{1}, \ldots, b_{n}\right)^{T}$,

then system (3) for $|\varepsilon| \neq 0$ sufficiently small has exactly one limit cycle bifurcating from the circle of radius $r_{0}$ centered at the origin of the plane $x_{3}=\cdots=x_{n}=0$, where $r_{0}$ is the unique solution in the interval $(1, \infty)$ of the equation

$$
\arctan \sqrt{r^{2}-1}-\frac{\sqrt{r^{2}-1}}{r^{2}}=\frac{\left(a_{11}+a_{22}+b_{1}\right) \pi}{2 b_{1}} .
$$

As far as we know there are almost no results as the one of Theorem 2, i.e. that provide explicit sufficient conditions for the existence of limit cycles in arbitrary dimension for piecewise linear differential systems of the form (3).

We emphasize that the bifurcation from $\varepsilon=0$ to $\varepsilon \neq 0$ in Theorem 2 takes place for $\varepsilon>0$ and for $\varepsilon<0$ sufficiently small, i.e. in both sides of the value $\varepsilon=0$. We remark that in a Hopf bifurcation the limit cycle only appears in one side of the bifurcation value of the parameter, but in our case which the limit cycle bifurcates from the period annulus of a center they appear in both sides of the parameter.

The proof of Theorem 2 is based on the first order averaging method. This method has been used frequently for computing periodic orbits, see for instance [12]. We will present this method in Section 2, in the form recently obtained in [4]. The advantage of this result is that the smoothness assumptions for the vector field of the differential system are minimal. In particular, it can be applied to piecewise linear differential systems, which are not $C^{2}$ (not even $C^{1}$ ), as it was required in its classical version, see for instance, Theorem 11.5 of Verhulst [15]. This non-differential application of the averaging method to control systems was used by first time in [5]. From the paper [6] we can study the stability of the limit cycle given in Theorem 2 for more details see Remark 7 .

The proof of Theorem 2 will be the subject of Section 3. The first step in the study of system (3), Lemma 1, is to reduce the number of parameters by a linear change of variables. The next objective is to transform the system into one which is in the standard form for averaging.

\section{First Order Averaging Method}

The aim of this section is to present the first order averaging method. See [4] for precise definitions and proofs. Differentiability of the vector field is not needed. The specific conditions for the existence of a simple isolated zero of the averaged function are given in terms of the Brouwer degree. In fact, the Brouwer degree theory is the key point in the proof of this theorem. We remind here that continuity of some finite dimensional 
function is a sufficient condition for the existence of its Brouwer degree (see [13] for precise definitions).

Theorem 3. We consider the following differential system

$$
\dot{x}(t)=\varepsilon H(t, x)+\varepsilon^{2} R(t, x, \varepsilon),
$$

where $H: \mathbb{R} \times D \rightarrow \mathbb{R}^{n}, R: \mathbb{R} \times D \times\left(-\varepsilon_{f}, \varepsilon_{f}\right) \rightarrow \mathbb{R}^{n}$ are continuous functions, $T$-periodic in the first variable, and $D$ is an open subset of $\mathbb{R}^{n}$. We define $h: D \rightarrow \mathbb{R}^{n}$ as

$$
h(z)=\frac{1}{T} \int_{0}^{T} H(s, z) d s,
$$

and assume that

(i) $H$ and $R$ are locally Lipschitz with respect to $x$;

(ii) for $p \in D$ with $h(p)=0$, there exists a neighborhood $V$ of $p$ such that $h(z) \neq 0$ for all $z \in \bar{V} \backslash\{p\}$ and the Brouwer degree $d_{B}(h, V, p) \neq 0$.

Then, for $|\varepsilon|>0$ sufficiently small, there exists an isolated T-periodic solution $\psi(\cdot, \varepsilon)$ of system (5) such that $\psi(\cdot, \varepsilon) \rightarrow p$ as $\varepsilon \rightarrow 0$.

Here we will need some facts from the proof of Theorem 3. Hypothesis (i) assures the existence and uniqueness of the solution of each initial value problem on the interval $[0, T]$. Hence, for each $z \in D$, it is possible to denote by $x(\cdot, z, \varepsilon)$ the solution of $(5)$ with the initial value $x(0, z, \varepsilon)=z$. We consider also the displacement function $\zeta: D \times\left(-\varepsilon_{f}, \varepsilon_{f}\right) \rightarrow \mathbb{R}^{n}$ defined by

$$
\zeta(z, \varepsilon)=\int_{0}^{T}\left[\varepsilon H(t, x(t, z, \varepsilon))+\varepsilon^{2} R(t, x(t, z, \varepsilon), \varepsilon)\right] d t .
$$

From the proof of Theorem 3 we extract the following facts.

Remark 4. Under the assumptions of Theorem 3 for every $z \in D$ the following relation holds for the displacement function

$$
\zeta(z, \varepsilon)=x(T, z, \varepsilon)-x(0, z, \varepsilon) .
$$

Moreover the displacement function $\zeta$ can be written in the form

$$
\zeta(z, \varepsilon)=\varepsilon h(z)+\varepsilon^{2} O(1)
$$

where $h$ is given by (6) and the symbol $O(1)$ denotes a bounded function on every compact subset of $D \times\left(-\varepsilon_{f}, \varepsilon_{f}\right)$. Moreover, for $|\varepsilon|$ sufficiently small, $p=\psi(0, \varepsilon)$ is an isolated zero of the displacement function $\zeta(\cdot, \varepsilon)$.

Note that from Remark 4 it follows that a zero $z$ of the displacement function $\zeta(z, \varepsilon)$ provides initial conditions for a periodic orbit of the system of period $T$. Consequently the zeros of $h(z)$ when $h(z)$ is not identically zero also provide periodic orbits of period $T$.

For a given system there is the possibility that the displacement function $\zeta$ is not globally differentiable, but the function $h$ is. In fact, only differentiability in some neighborhood of a fixed isolated zero of $h$ could be enough. 
When this is the case, one can use the following remark in order to verify the hypothesis $(i i)$ of Theorem 3.

Remark 5. Let $h: D \rightarrow \mathbb{R}^{n}$ be a $C^{1}$ function, with $h(p)=0$, where $D$ is an open subset of $\mathbb{R}^{n}$ and $p \in D$. Whenever $p$ is a simple zero of $h$ (i.e. the Jacobian of $h$ at $p$ is not zero), then there exists a neighborhood $V$ of $p$ such that $h(z) \neq 0$ for all $z \in \bar{V} \backslash\{p\}$. Then the Brouwer degree $d_{B}(h, V, p) \in\{-1,1\}$.

\section{Proof of Theorem 2}

The proof of Theorem 2 is based on the first order averaging method presented in the previous section. In order to apply this result, we will prove first that it is possible to reduce the number of parameters and after we will change the variables in order to transform the system into one which is in the standard form for averaging. The computation of the averaged function (6) will be also a special task. After we must determine the number of its isolated zeros. The relation between the averaging method and the displacement function will be also discussed.

Proof of Lemma 1. Since the linear change of variables $x=J y$, with $J$ an invertible matrix, transforms system (1) into

$$
y^{\prime}=J^{-1} A_{0} J y+\varepsilon J^{-1} A J y+\varepsilon \varphi\left(k^{T} J y\right) J^{-1} b,
$$

we have to find $J$ such that

$$
J^{-1} A_{0} J=A_{1}, \quad k^{T} J=e_{1}^{T} .
$$

To solve the previous two equations for obtaining the matrix $J$ is equivalent to solve the system

$$
J^{-1} A_{0}=A_{1} J^{-1}, \quad k^{T}=e_{1}^{T} J^{-1} .
$$

Easy computations show that the matrix

$$
J^{-1}=\left(\begin{array}{cc}
k_{1} & k_{2} \\
-k_{2} & k_{1}
\end{array}\right)
$$

if $n=2$, and

$$
J^{-1}=\left(\begin{array}{ccccccc}
k_{1} & k_{2} & k_{3} & k_{4} & k_{5} & \ldots & k_{n} \\
-k_{2} & k_{1} & -k_{3} & -k_{4} & -k_{5} & \cdots & -k_{n} \\
0 & 0 & -k_{3} \varepsilon^{-1} & -k_{4} \varepsilon^{-1} & -k_{5} \varepsilon^{-1} & \cdots & -k_{n} \varepsilon^{-1} \\
0 & 0 & 0 & 1 & 0 & \cdots & 0 \\
0 & 0 & 0 & 0 & 1 & \cdots & 0 \\
\vdots & \vdots & \vdots & \vdots & \vdots & \cdots & \vdots \\
0 & 0 & 0 & 0 & 0 & \cdots & 1
\end{array}\right)
$$

if $n>2$, satisfies the two equations (8). Since the determinant of $J^{-1}$ is $k_{1}^{2}+k_{2}^{2} \neq 0$ if $n=2$, and $-\left(k_{1}^{2}+k_{2}^{2}\right) k_{3} / \varepsilon$ if $n>2$, there exists the change of variables $x=J y$ proving the lemma. 
An equivalent system to system (3), which is in the standard form for averaging, will be obtained now by a proper change of the variables. Thus changing the variables $\left(x_{1}, x_{2}, x_{3}, \ldots, x_{n}\right)$ to $\left(r, \theta, x_{3}, \ldots, x_{n}\right)$ by $x_{1}=r \cos \theta$ and $x_{2}=r \sin \theta$, system (3) is transformed into a system of the form

$$
\frac{d r}{d \theta}=\varepsilon H_{1}\left(\theta, r, x_{3}, \ldots, x_{n}\right)+\varepsilon^{2} O(1)
$$

$$
\frac{d x_{j}}{d \theta}=\varepsilon H_{j}\left(\theta, r, x_{3}, \ldots, x_{n}\right)+\varepsilon^{2} O(1),
$$

for $j=3, \ldots, n$, where

$$
\begin{aligned}
H_{1}= & \cos \theta\left[a_{11} r \cos \theta+a_{12} r \sin \theta+\sum_{k=3}^{n} a_{1 k} x_{k}+b_{1} \varphi(r \cos \theta)\right]+ \\
& \sin \theta\left[a_{21} r \cos \theta+a_{22} r \sin \theta+\sum_{k=3}^{n} a_{2 k} x_{k}+b_{2} \varphi(r \cos \theta)\right], \\
H_{j}= & a_{j 1} r \cos \theta+a_{j 2} r \sin \theta+\sum_{k=3}^{n} a_{j k} x_{k}+b_{j} \varphi(r \cos \theta) .
\end{aligned}
$$

to system (9). Here $\bar{A}=\left(a_{i j}\right)$ if $n=2$, and $a_{i j}$ is the element of the row $i$ and column $j$ of the $n \times n$ matrix

$$
\bar{A}+\left(\begin{array}{cccccc}
0 & 0 & \varepsilon & 0 & \cdots & 0 \\
0 & 0 & \varepsilon & 0 & \cdots & 0 \\
0 & 0 & 0 & 0 & \cdots & 0 \\
\vdots & \vdots & \vdots & \vdots & \cdots & \vdots \\
0 & 0 & 0 & 0 & \cdots & 0
\end{array}\right)
$$

if $n>2$.

Our next step is to find the corresponding function (6), so we must compute

$$
h_{i}\left(r, x_{3}, \ldots, x_{n}\right)=\frac{1}{2 \pi} \int_{0}^{2 \pi} H_{i}(\theta, r, \rho, s) d \theta,
$$

for $i=1, \ldots, n-1$.

For each $r>0$ we denote

$$
\begin{aligned}
& I_{0}(r)=\int_{0}^{2 \pi} \varphi(r \cos \theta) d \theta, \\
& I_{1}(r)=\int_{0}^{2 \pi} \varphi(r \cos \theta) \cos \theta d \theta, \\
& I_{2}(r)=\int_{0}^{2 \pi} \varphi(r \cos \theta) \sin \theta d \theta,
\end{aligned}
$$

where $\varphi$ is the piecewise-linear function given by (2). 
Lemma 6. The integrals $I_{0}, I_{1}$ and $I_{2}$ given by (11), (12) and (13) respectively, have the following expressions

$$
I_{0}(r)=0, \text { for all } r>0
$$

$$
I_{1}(r)= \begin{cases}r \pi, & \text { for } 0<r \leq 1, \\ 2 \frac{\sqrt{r^{2}-1}}{r}+r \pi-2 r \arctan \sqrt{r^{2}-1}, & \text { for } r>1 ;\end{cases}
$$

and

$$
I_{2}(r)=0, \text { for all } r>0 .
$$

Proof. Whenever $0<r \leq 1$ we have that $|r \cos \theta| \leq 1$ and $|r \sin \theta| \leq 1$ for all $\theta \in[0,2 \pi)$. Then $\varphi(r \cos \theta)=r \cos \theta$ for every $\theta$. Thus

$$
\begin{aligned}
& I_{0}(r)=r \int_{0}^{2 \pi} \cos \theta d \theta=0, \\
& I_{1}(r)=r \int_{0}^{2 \pi} \cos ^{2} \theta d \theta=r \pi, \\
& I_{2}(r)=r \int_{0}^{2 \pi} \sin \theta \cos \theta d \theta=0 .
\end{aligned}
$$

We fix now $r>1$ and consider $\theta_{c} \in(0, \pi / 2)$ such that $\cos \theta_{c}=1 / r$. Then we can write

$$
\begin{aligned}
I_{0}(r)= & \int_{0}^{\theta_{c}} d \theta+r \int_{\theta_{c}}^{\pi-\theta_{c}} \cos \theta d \theta-\int_{\pi-\theta_{c}}^{\pi+\theta_{c}} d \theta+ \\
& r \int_{\pi+\theta_{c}}^{2 \pi-\theta_{c}} \cos \theta d \theta+\int_{2 \pi-\theta_{c}}^{2 \pi} d \theta \\
I_{1}(r)= & \int_{0}^{\theta_{c}} \cos \theta d \theta+r \int_{\theta_{c}}^{\pi-\theta_{c}} \cos ^{2} \theta d \theta-\int_{\pi-\theta_{c}}^{\pi+\theta_{c}} \cos \theta d \theta+ \\
& r \int_{\pi+\theta_{c}}^{2 \pi-\theta_{c}} \cos ^{2} \theta d \theta+\int_{2 \pi-\theta_{c}}^{2 \pi} \cos \theta d \theta,
\end{aligned}
$$

and

$$
\begin{aligned}
I_{2}(r)= & \int_{0}^{\theta_{c}} \sin \theta d \theta+r \int_{\theta_{c}}^{\pi-\theta_{c}} \sin \theta \cos \theta d \theta-\int_{\pi-\theta_{c}}^{\pi+\theta_{c}} \sin \theta d \theta+ \\
& r \int_{\pi+\theta_{c}}^{2 \pi-\theta_{c}} \sin \theta \cos \theta d \theta+\int_{2 \pi-\theta_{c}}^{2 \pi} \sin \theta d \theta .
\end{aligned}
$$

Straightforward computations lead to the following expressions

$$
I_{0}(r)=0, \quad I_{1}(r)=2 \sin \theta_{c}+r \pi-2 r \theta_{c}, \quad \text { and } \quad I_{2}(r)=0 .
$$

Since $\theta_{c} \in(0, \pi / 2)$ with $\cos \theta_{c}=1 / r$, we have $\sin \theta_{c}=\sqrt{r^{2}-1} / r$ and $\theta_{c}=\arctan \sqrt{r^{2}-1}$. 
We note that the functions $I_{i}(r)$ for $i=1,2$ already appeared in [4]. In short from (10) we get that $h_{1}\left(r, x_{3}, \ldots, x_{n}\right)=-\frac{b_{1}}{\pi} r \arctan \sqrt{r^{2}-1}+\frac{b_{1}}{\pi r} \sqrt{r^{2}-1}+\frac{1}{2}\left(a_{11}+a_{22}+b_{1}\right) r$,
$h_{j}\left(r, x_{3}, \ldots, x_{n}\right)=a_{j 3} x_{3}+\ldots+a_{j n} x_{n}$,

for $j=2, \ldots, n-1$.

In order that the system $h_{i}\left(r, x_{3}, \ldots, x_{n}\right)=0$ for $i=1, \ldots, n-1$ has isolated solutions, otherwise the Jacobian on them become zero and we cannot apply Theorem 3 for studying the limit cycles of system (3) for $|\varepsilon| \neq 0$ sufficiently small, it follows for $n>2$ that the determinant of the matrix $B$ obtained from the minor of the matrix $\bar{A}$ erasing the first two rows and the first two columns is not zero. Then from the equations $h_{i}\left(r, x_{3}, \ldots, x_{n}\right)=$ 0 for $i=2, \ldots, n-1$ we get that $x_{3}=\cdots=x_{n}=0$. From equation $h_{1}\left(r, x_{3}, \ldots, x_{n}\right)=0$ we have

$$
f(r)=\arctan \sqrt{r^{2}-1}-\frac{\sqrt{r^{2}-1}}{r^{2}}=\frac{\left(a_{11}+a_{22}+b_{1}\right) \pi}{2 b_{1}} .
$$

Since the function $f:(1, \infty) \rightarrow(0, \pi / 2)$ is a diffeomorphism, Theorem 2 follows directly of Theorem 3 if the Jacobian

$$
\operatorname{det}\left(\left.\frac{\partial\left(h_{1}, \ldots, h_{n-1}\right)}{\partial\left(r, x_{3}, \ldots, x_{n}\right)}\right|_{\left(r, x_{3}, \ldots, x_{n}\right)=\left(r_{0}, 0, \ldots, 0\right)}\right) \neq 0
$$

where $r_{0}$ is the unique solution of $f\left(r_{0}\right)=\left(a_{11}+a_{22}+b_{1}\right) \pi /\left(2 b_{1}\right) \in(0, \pi / 2)$.

It is easy to check that (17) is equal to

$$
\frac{2 b_{1} \operatorname{det}(B) \sqrt{r_{0}^{2}-1}}{\pi r_{0}^{2}} \neq 0,
$$

because $r_{0}>1$ and we are assuming that $\operatorname{det}(B) \neq 0$ and $\left(a_{11}+a_{22}+\right.$ $\left.b_{1}\right) /\left(2 b_{1}\right) \in(0,1 / 2)$. In short Theorem 2 is proved.

Remark 7. Using the main result of [6] the stability of the limit cycle associated to the solution $\left(r_{0}, 0, \ldots, 0\right)$ is given by the eigenvalues of the matrix

$$
\left.\frac{\partial\left(h_{1}, \ldots, h_{n-1}\right)}{\partial\left(r, x_{3}, \ldots, x_{n}\right)}\right|_{\left(r, x_{3}, \ldots, x_{n}\right)=\left(r_{0}, 0, \ldots, 0\right)} .
$$

Acknowledgements. We thank to the referee his comments which helps us to improve the first version of this paper.

\section{REFERENCES}

[1] M.A. Aizerman, Theory of automatic control, Pergamon Press, 1963.

[2] D.P. Atherton, Nonlinear control engineering (Describing function analysis and design), 2nd edition, Van Nostrand Reinhold, New York 1982.

[3] S. BarnetT AND R.G. CAmeron, Introduction to mathematical control theory, 2nd edition, Clarendon Press, Oxford 1985. 
[4] A. Buică And J. Llibre, Averaging methods for finding periodic orbits via Brouwer degree, Bull. Sci. Math. 128 (2004), 7-22.

[5] A. Buiç̆ AND J. LliBRe, Bifurcation of limit cycles from a 4-dimensional center in control systems, Int. J. Bif. Chaos 15 (2004), 2653-2662.

[6] A. Buica, J. Llibre And O. Makarenkov, Asymptotic stability of periodic solutions for nonsmooth differential equations with application to the nonsmooth van der Pol oscillator, SIAM J. of Math. Anal. 40 (2009), 2478-2495.

[7] S.N. Chow And J. Hale, Methods of bifurcation theory, Springer-Verlag New York, Heidelberg, Berlin, 1982.

[8] J.E. Gibson, Nonlinear automatic control, McGraw-Hill, New York 1963.

[9] J. Guckenheimer AND P. Holmes, Nonlinear oscillations, dynamical systems, and bifurcation of vector fields, Springer-Verlag New York, Heidelberg, Berlin, 1983.

[10] Chengzhi Li, Abelian integrals and applications to weak Hilbert's $16^{\text {th }}$ problem, in "Limit cycles of differential equations", C. Christopher and C. Li, pp. 91-162, Advanced Courses in Math. CRM, Barcelona, Birkhäuser, Basel, 2007.

[11] H.K. KhaliL, Nonlinear systems, Macmillan, New York 1992.

[12] J. Llibre, J.S. PÉRez DEL Río AND J.A. Rodríguez, Averaging analysis of a perturbated quadratic center, Nonlinear Anal. 46 (2001) Ser. A: Theory Methods, $45-51$.

[13] N.G. Lloyd, Degree Theory, Cambridge University Press, 1978.

[14] A.I. MeEs, Dynamics of feedback systems, John Wiley \& Sons 1981.

[15] F. Verhulst, Nonlinear Differential Equations and Dynamical Systems, Second Edition, Universitext, Springer, 1996.

[16] M. Vidyasagar, Nonlinear systems analysis 2nd ed., Prentice-Hall 1993.

Departament de Matemàtiques, Universitat Autònoma de Barcelona, 08913 Bellaterra, Barcelona, Spain

E-mail address: jllibre@mat.uab.cat

Department of mathematics, Faculty of Sciences, University of Annaba, BP12, Sidi Amar, Annaba, Algeria

E-mail address: makhloufamar@yahoo.fr 